\title{
Trichloroacetimidate-Triggered Expeditious and Novel Synthesis of $\mathrm{N}$-Acylbenzotriazoles
}

\author{
Mangal S. Yadav \\ Manoj K. Jaiswal \\ Sunil Kumar \\ Vinod K. Tiwari* (D)
}

Department of Chemistry, Institute of Science, Banaras Hindu University, Varanasi, 221005, India

tiwari_chem@yahoo.co.in

Vinod.Tiwari@bhu.ac.in

This manuscript is dedicated to Prof. (em). Richard R. Schmidt for his notable contribution on imidate chemistry

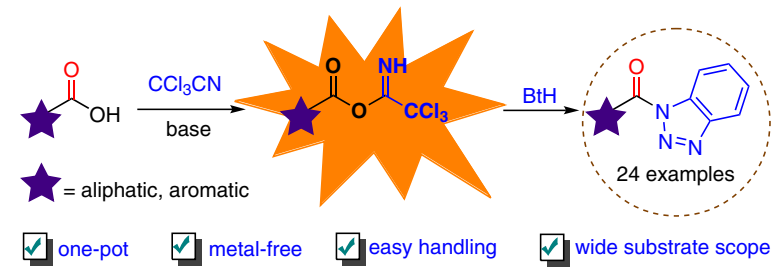

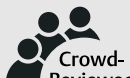

Crowd-

Accepted after revision: 27.09 .2021

Published online: 27.09 .2021

DOI: 10.1055/a-1656-7293; Art ID: so-2021-d0045-op

License terms: $c$ ( $) \$$

(c) 2021. The Author(s). This is an open access article published by Thieme under the terms of the Creative Commons Attribution-NonDerivative-NonCommercial-License, permitting copying and reproduction so long as the original work is given appropriate credit. Contents may not be used for commercial purposes or adapted, remixed, transformed or built upon. (https://creativecommons.org/licenses/by-nc-nd/4.0/)

Abstract A facile route for the synthesis of a diverse range of $\mathrm{N}$-acylbenzotriazole derivatives from the corresponding carboxylic acids has been established through a carbonyl activation pathway. In this method, trichloroacetonitrile is performed as an effective reagent for an easy access of $\mathrm{N}$-acylbenzotriazoles which was simply proceeded through the activation of carboxylic acids via in situ imidate formation in anhydrous 1,2-dichloroethane followed by addition of $1 \mathrm{H}$-benzotriazole at $80^{\circ} \mathrm{C}$ for 3-4 h. Easy handling, one-pot, and metal-free conditions demonstrate the notable merits of the devised protocol.

Keywords $\mathrm{N}$-acylbenzotriazole, benzotriazole, coupling reaction, imidate, trichloroacetonitrile

The benzotriazole methodology successfully offers a versatile synthetic protocol in organic synthesis for the rapid construction of a diverse range of molecular architectures having robust applications in chemistry, biology, and material science. ${ }^{1,2}$ The notable advantages, such as the nontoxic, inexpensive, and high stability nature of benzotriazole make it a useful synthetic auxiliary. Toward this end, numerous benzotriazole-based reagents, ligands, and intermediates have received attention in several organic transformations, mainly because of their special chemical behavior during the course of reaction. ${ }^{3-5}$ Various reports have been documented for the synthesis of esters and amides, ${ }^{6}$ thioesters, ${ }^{7}$ acyl azides, ${ }^{8}$ peptides, ${ }^{9}$ diketones, ${ }^{10} \beta$-ketonitriles, ${ }^{11}$ sulfones, ${ }^{12}$ oxazolines, and thiazolines, ${ }^{13}$ using $\mathrm{N}$-acylbenzotriazoles through $\mathrm{N}$-, $\mathrm{O}-, \mathrm{S}$-, and C-acylations under ambient conditions. ${ }^{14}$ Furthermore, benzotriazole ring cleavage (BtRC) methodology enables the synthesis of $\mathrm{N}$-phenylamides, ${ }^{15}$ benzoxazoles, ${ }^{16}$ and benzothiazoles, ${ }^{17}$ significantly by utilizing the respective $\mathrm{N}$-acylbenzotriazoles. In addition, carbamates, ureas, and thiocarbamates have been successfully prepared through Curtius rearrangement in good-to-excellent yields. ${ }^{18}$ Therefore, development of novel and common routes for an easy access of $\mathrm{N}$-acylbenzotriazoles is a highly promising area.

The formation of $\mathrm{N}$-acylbenzotriazoles involves the key transformation of carboxylic acids in organic synthesis. Therefore, efforts have been devoted to develop robust protocols for easy access to $\mathrm{N}$-acylbenzotriazoles (Scheme 1 ). The approaches are commonly characterized by two archetypal pathways. The first involves carbonyl activation (where BtH is added to activated carboxylate), which mainly comprises activators such as $\mathrm{I}_{2} / \mathrm{PPh}_{3}, \mathrm{NBS} / \mathrm{PPh}_{3}$, or $\mathrm{TCT} / \mathrm{PPh}_{3},{ }^{19-22} \mathrm{EDC}$ or $\mathrm{DCC}^{23}$ along with tosyl chloride ${ }^{24}$ and BtH with or without base. Alternatively, activation of benzotriazole involves a carboxylic acid being added to the activated BtH. Surprisingly, very few reports are available for the $\mathrm{BtH}$ activation process. For example, 1-(methane sulfonyl)benzotriazole has been shown to be electrophilic enough to react with carboxylic acids in the presence of $\mathrm{Et}_{3} \mathrm{~N}$, ${ }^{25 \mathrm{a}}$ and the reaction of thionyl chloride with $\mathrm{BtH}$ (3-4 equiv.) accomplished the acylation of benzotriazole. ${ }^{25 b}$ Investigations of suitable reagents that proceed through $\mathrm{BtH}$ activation has received less attention because of the side products generated from the loss of the leaving group are reactive towards sensitive functionalities. Moreover, the carbonyl activation pathway has been explored, mainly because this process avoids the use of strong acids and bases which makes the transformation more convenient and practical. We have recently reported a new approach using 2,2'-dipyridyl disulfide/ $\mathrm{PPh}_{3}{ }^{26}$ and further extended this with the aid of TCICA/PPh ${ }_{3}{ }^{27}$ for easy access to diverse $\mathrm{N}$-acylbenzotriazoles from the corresponding acids through a 
carbonyl activation pathway in high-to-excellent yields. In the context of carbonyl activation, a report is well documented for the conversion of carboxylic acids into respective amides (via acid chloride formation) using the combination of the activators trichloroacetonitrile (TCA) and $\mathrm{PPh}_{3}{ }^{28}$ However, the disclosed protocol was limited to the synthesis of benzamides, rather than acylation of benzotriazole. ${ }^{28}$

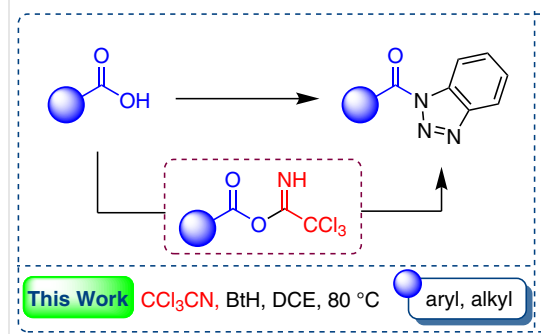

Previous Methods

Method 1

(a) PySSPy, $\mathrm{PPh}_{3}, \mathrm{BtH}, \mathrm{DCM}$ (b) TCICA, $\mathrm{PPh}_{3}, \mathrm{BtH}, \mathrm{DCM}$, (c) $\mathrm{NBS}, \mathrm{PPh}_{3}, \mathrm{BtH}, \mathrm{DCM}$ (d) $\mathrm{I}_{2}, \mathrm{PPh}_{3}, \mathrm{BtH}, \mathrm{Et}_{3} \mathrm{~N}, \mathrm{DCM}$ (e) EDC or DCC, BtH, DCM Method 2

(c) $\mathrm{BtH} / \mathrm{SOCl}_{2}(4: 1), \mathrm{DCM}$ (d) $\mathrm{BtSO}_{2} \mathrm{R}, \mathrm{Et}_{3} \mathrm{~N}, \mathrm{THF}$, reflux

Scheme 1 Established tactics for synthesis of $\mathrm{N}$-acylbenzotriazoles from the corresponding carboxylic acids.

Functionalization of various groups via imidate formation is well known, and they are the important class of intermediates to introduce different groups, ${ }^{29}$ as well as acting as a directing group. ${ }^{30}$ Different imidate-based complexes of gold ${ }^{31}$ and palladium ${ }^{32}$ are also known for use in catalysis. Among which, trichloroacetimidates are known to be powerful leaving groups in glycoscience ${ }^{33-36}$ and have been extensively explored as glycosyl donors in glycosylation reactions with various acceptors in the presence of a suitable promoter.$^{37}$ Numerous reports have demonstrated the formation of $\mathrm{O} / \mathrm{N}$-glycosides with glycosyl trichloroacetimidates that do not require the addition of a promoter. ${ }^{38}$

Thus, in continuation of our previous studies into $\mathrm{N}$-acylbenzotriazole synthesis, we have continued investigating the activation of carboxylic acids via trichloroacetimidates and have established that these act as effective reagents for easy access to $\mathrm{N}$-acylbenzotriazoles, the results of which we wish to report herein.

Our investigation commenced with a model reaction of 1-naphthoic acid (1.0 equiv.) with trichloroacetonitrile $\left(\mathrm{CCl}_{3} \mathrm{CN}, 1.0\right.$ equiv.) and 4-dimethylaminopyridine (DMAP, 0.5 equiv.) in dichloromethane (DCM) at $60{ }^{\circ} \mathrm{C}$ for $2.0 \mathrm{~h}$ to afford (1H-benzo[d][1,2,3]triazol-1-yl)(napthalen-1-yl)methanone (2q) in moderate yield (Table 1, entry 1 ). After establishing $\mathrm{CCl}_{3} \mathrm{CN}$ as suitable reagent, we proceeded to improve the reaction yield. The types of base and $\mathrm{CCl}_{3} \mathrm{CN}$ in various ratios and solvents were investigated at different temperatures and for the different time intervals. The yield was seen to be enhanced when 1.0 equivalent of base is used at $80^{\circ} \mathrm{C}$ for $3 \mathrm{~h}$ under the established reaction conditions (Table 1 , entry 2 ). In continuation, the conversion was improved when the molar ratio of $\mathrm{CCl}_{3} \mathrm{CN}$ was doubled at $80^{\circ} \mathrm{C}$ for $3 \mathrm{~h}$ in DCE, resulting in $80 \%$ yield of compound $\mathbf{2 q}$ (Table 1, entry 4). Increasing the amount of $\mathrm{CCl}_{3} \mathrm{CN}$ up to
4.0 equivalents did not result in a better outcome in terms of reaction yield (Table 1 entry 5). However, no product was detected when the reaction was carried without $\mathrm{CCl}_{3} \mathrm{CN}$ (Table 1, entry 6), confirming that $\mathrm{CCl}_{3} \mathrm{CN}$ is essential. Towards optimization, we examined the reaction by varying the solvents such as toluene, $\mathrm{CHCl}_{3}$, and DMF (Table 1, entries 7-9), although these were found to be less efficient than DCE. Water was also taken examined, but no conversion was observed (Table 1, entry 10). Different bases such as $\mathrm{Et}_{3} \mathrm{~N}, \mathrm{DBU}$, and $\mathrm{K}_{2} \mathrm{CO}_{3}$ were considered, but no reasonable improvement in the yield was detected. Among these only DBU was found to be efficient, which showed approximately the same result as DMAP (Table 1, entry 12). The reaction was also carried out without base (Table 1, entry 14), but only traces of product were detected, which confirms that base is necessary. At room temperature, low conversion was observed (Table 1, entry 15), but conversion increases with increasing temperature up to $80^{\circ} \mathrm{C}$ (Table 1 , entry 4 ) and a notable reduction in yield was observed when the reaction was carried out at higher temperature (Table 1, entries 16 and 17). From the above investigations we concluded that

Table 1 Reaction Optimization Study for N-Acylbenzotriazole Synthesis via Trichloroacetimidate Intermediates

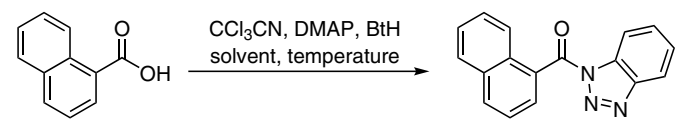

$1 \mathrm{q}$

$2 q$

\begin{tabular}{|c|c|c|c|c|c|c|}
\hline Entry ${ }^{a}$ & $\begin{array}{l}\mathrm{CCl}_{3} \mathrm{CN} \\
\text { (equiv.) }\end{array}$ & $\begin{array}{l}\text { Temp } \\
\left({ }^{\circ} \mathrm{C}\right)\end{array}$ & Base (equiv.) & Solvent $\mathrm{t}^{\mathrm{b}}$ & $\begin{array}{l}\text { Time } \\
\text { (h) }\end{array}$ & $\begin{array}{l}\text { Yield } \\
(\%)^{c}\end{array}$ \\
\hline 1 & 1.0 & 60 & DMAP $(0.5)$ & DCM & 2 & 45 \\
\hline 2 & 1.0 & 80 & DMAP (1.0) & DCM & 3 & 50 \\
\hline 3 & 1.0 & 80 & $\operatorname{DMAP}(1.0)$ & DCE & 3 & 58 \\
\hline 4 & 2.0 & 80 & DMAP (1.0) & DCE & 3 & 80 \\
\hline 5 & 4.0 & 80 & DMAP (1.0) & DCE & 3 & 78 \\
\hline 6 & - & 80 & DMAP (1.0) & DCE & 3 & NIL \\
\hline 7 & 2.0 & 80 & $\operatorname{DMAP}(1.0)$ & toluene & 3 & 70 \\
\hline 8 & 2.0 & 80 & DMAP (1.0) & DMF & 3 & 45 \\
\hline 9 & 2.0 & 80 & DMAP (1.0) & $\mathrm{CHCl}_{3}$ & 3 & 52 \\
\hline 10 & 2.0 & 80 & DMAP (1.0) & $\mathrm{H}_{2} \mathrm{O}$ & 8 & NIL \\
\hline 11 & 2.0 & 80 & $\mathrm{Et}_{3} \mathrm{~N}(1.0)$ & DCE & 3 & 42 \\
\hline 12 & 2.0 & 80 & $\mathrm{DBU}(1.0)$ & DCE & 3 & 70 \\
\hline 13 & 2.0 & 80 & $\mathrm{~K}_{2} \mathrm{CO}_{3}(1.0)$ & DCE & 3 & 48 \\
\hline 14 & 2.0 & 80 & - & DCE & 3 & 40 \\
\hline 15 & 2.0 & r.t & $\operatorname{DMAP}(1.0)$ & DCE & 3 & trace \\
\hline 16 & 2.0 & 120 & DMAP (1.0) & DCE & 3 & 78 \\
\hline 17 & 2.0 & 150 & DMAP (1.0) & DCE & 3 & 72 \\
\hline
\end{tabular}

a Reactions carried out at reflux under argon atmosphere.

${ }^{\mathrm{b}}$ Dry solvents were used.

c Yields reported after column chromatography $\left(\mathrm{SiO}_{2}\right)$. 
optimal conditions involved $\mathrm{CCl}_{3} \mathrm{CN}$ (2.0 equiv.) and DMAP (1.0 equiv.) in DCE, followed by the addition of $1 H$-benzotriazole at $80^{\circ} \mathrm{C}$ for $3 \mathrm{~h}$. Under these conditions, compound $\mathbf{2 q}$ was isolated in $80 \%$ yield after column chromatography (Table 1 , entry 4 ).

Subsequently the optimized protocol was applied to construct a library of diverse $\mathrm{N}$-acylbenzotriazole derivatives 2a-t by incorporation of various substituents on the benzene ring of the carboxylic acids (Scheme 2). Furthermore, we investigated the effect of substituents on the ben-

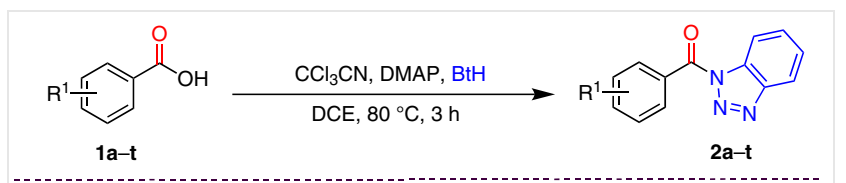<smiles>O=C(c1ccc(Cl)cc1)n1nnc2ccccc21</smiles>

2a (70\%)<smiles>O=C(c1ccccc1Cl)n1nnc2ccccc21</smiles>

2b (68\%)<smiles>O=C(c1cccc(Cl)c1Cl)n1nnc2ccccc21</smiles>

2d (57\%)<smiles>O=C(c1ccccc1Br)n1nnc2ccccc21</smiles>

2g (67\%)<smiles>O=C(c1ccccc1F)n1nnc2ccccc21</smiles>

2j (52\%)<smiles>Cc1cccc(C(=O)n2nnc3ccccc32)c1</smiles>

$2 \mathrm{~m}(70 \%)$<smiles>O=C(c1ccccc1)n1nnc2ccccc21</smiles>

$2 p(75 \%)$

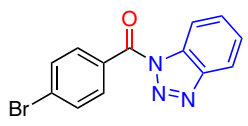

2 e $(68 \%)$<smiles>O=C(c1cc(Br)ccc1Br)n1nnc2ccccc21</smiles>

2h (60\%)<smiles>N#Cc1cccc(C(=O)n2nnc3ccccc32)c1</smiles>

2k (62\%)<smiles>Cc1cc(C)cc(C(=O)n2nnc3ccccc32)c1</smiles>

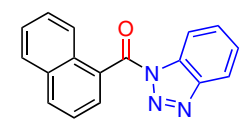

$2 q(80 \%)$

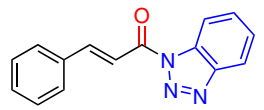

2t $(59 \%)$

Scheme 2 Molar ratios: substituted benzoic acids 1 (1.0 equiv.), $\mathrm{CCl}_{3} \mathrm{CN}$ (2.0 equiv.), DMAP (1.0 equiv.), BtH (1.0 equiv.). Yields reported after column chromatography $\left(\mathrm{SiO}_{2}\right)$.

2i $(57 \%)$

2l $(71 \%)$

$20(71 \%)$

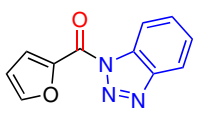

$2 \mathbf{r}(63 \%)$ zene ring of the carboxylic acids and found that the rings substituted with electron-withdrawing groups afforded the

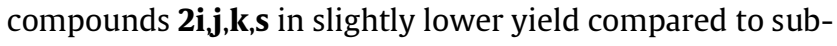

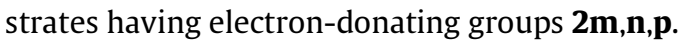

The reaction also proceeded well with polynuclear aromatic precursors. For example, the reaction of $\alpha$-naphthoic acid under the optimized conditions furnished a good yield of the respective $1 H$-benzo[d][1,2,3]triazol-1-yl)(napthalen-1-yl)methanone (2q). The $\alpha, \beta$-unsaturated acid, cinnamic acid, also reacted well to afford $(E)-1-\{1 H$-benzo[d][1,2,3]triazol-1-yl\}-3-phenylprop-2-en-1-one (2t) after column chromatography.

To explore the scope of the protocol, we considered the reaction with different aliphatic carboxylic acid derivatives under optimized conditions, where the respective $\mathrm{N}$-acylbenzotriazoles 3a-d were isolated after column chromatography in moderate to good yields (Scheme 3 ). The reaction was also carried out with short-chain aliphatic carboxylic acids such as acetic acid under optimized reaction protocol; unfortunately, no reaction was observed.
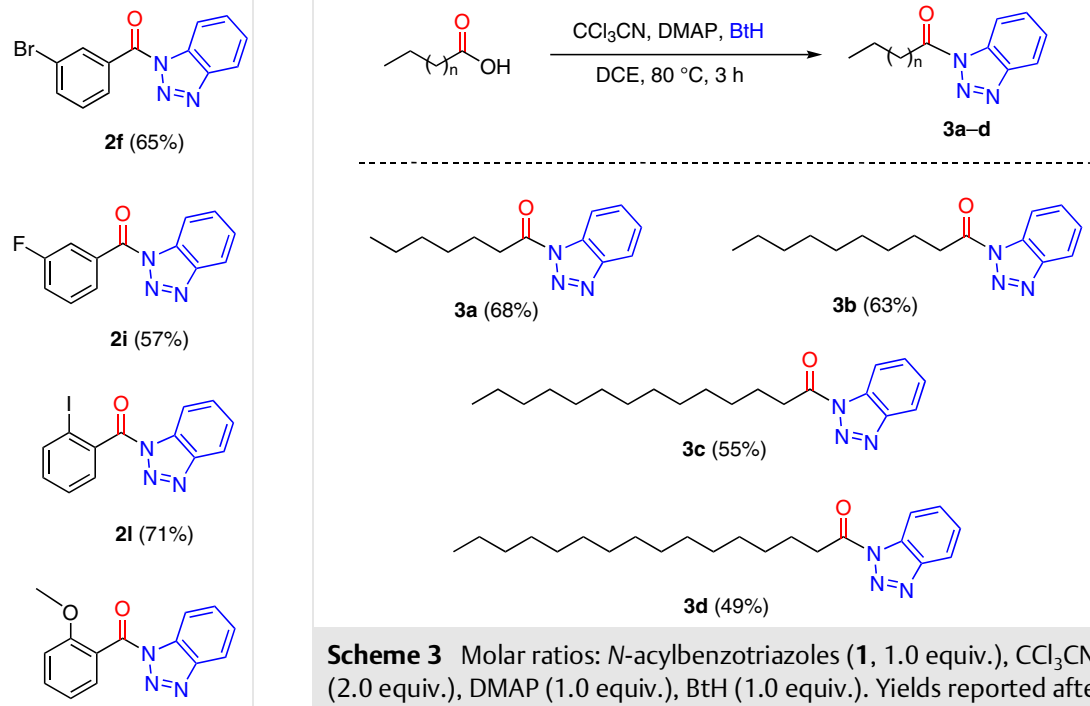

Scheme 3 Molar ratios: $N$-acylbenzotriazoles (1, 1.0 equiv.), $\mathrm{CCl}_{3} \mathrm{CN}$ (2.0 equiv.), DMAP (1.0 equiv.), BtH (1.0 equiv.). Yields reported after column chromatography $\left(\mathrm{SiO}_{2}\right)$.

Trichloroacetimidate as an electrophile has been utilized for esterification reactions under mild reaction conditions. ${ }^{39}$ This method involves activation of the alcohol, ${ }^{39}$ rather than activation of the carboxylic acid via trichloroacetimidate as described herein.

A possible mechanism for the preparation of $\mathbf{2}$ is proposed in Scheme 4 for which carboxylic acid $\mathbf{1}$ initially reacts with trichloroacetonitrile to give the corresponding trichloroacetimidic anhydride intermediate I. Subsequent, addition of benzotriazole to the intermediate II results in formation of final product $\mathbf{2}$ with the loss of trichloroacetamide III. 

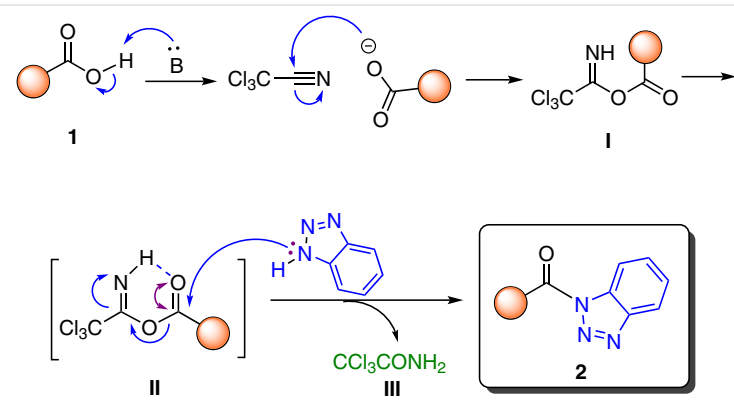

Scheme 4 Proposed mechanism for the synthesis of $\mathrm{N}$-acylbenzotriazole $\mathbf{2}$ via an intermediate imidate.

In conclusion, we report a facile route for the synthesis of $\mathrm{N}$-acylbenzotriazole derivatives by activation of different aromatic and aliphatic carboxylic acid derivatives with trichloroacetonitrile $\left(\mathrm{CCl}_{3} \mathrm{CN}\right)$. The reaction is proposed to proceed through the formation of the corresponding imidate intermediates leading to the formation of respective $\mathrm{N}$-acylbenzotriazole derivatives. The methodology is a onepot protocol with broad substrate scope.

All chemicals and solvents were of analytical grade. Thin-layer chromatography (TLC) was performed on 60 F254 silica gel, pre-coated on aluminium plates, and seen under a UV lamp $\left(\lambda_{\max }=254 \mathrm{~nm}\right)$. Solvents were condensed under low pressure at temperature $<55^{\circ} \mathrm{C}$. Column chromatography was subjected to silica gel (100-200 mesh, 230-400 mesh, Merck). Ethyl acetate and $n$-hexane were distilled before column chromatography. ${ }^{1} \mathrm{H},{ }^{13} \mathrm{C}$, and ${ }^{19} \mathrm{~F}$ NMR spectra were recorded at 500, 125, and $470 \mathrm{MHz}$, respectively. Chemical shifts were recorded in ppm downfield from internal TMS, $J$ values in $\mathrm{Hz}$. Mass spectra were recorded using SCIEX X500r Q-TOF, ahigh-resolution mass spectrometer (HRMS).

\section{Typical Experimental Procedure for the Synthesis of $\mathbf{N}$-Acylben- zotriazoles 2}

To a solution of carboxylic acid (1.0 equiv.) 1,2 dichloroethane, trichloroacetonitrile (2.0 equiv.), and DMAP (1.0 equiv.) were added. Then, the reaction mixture was shaken for $10 \mathrm{~min}$ at room temperature, followed by addition of $1 \mathrm{H}$-benzotriazole. The reaction was left at $80{ }^{\circ} \mathrm{C}$ for $3-4 \mathrm{~h}$. After the completion of reaction (monitored by TLC), the resulting reaction mixture was evaporated under reduced pressure. The residue was subjected to column chromatography (2$10 \%$ ethyl acetate $/ n$-hexane) to afford the desired $N$-acylbenzotriazoles.

\section{Physical Data for Aromatic and Aliphatic $N$-Acylbenzotriazoles 2a-t and 3a-d}

\section{(1H-Benzo[d] [1,2,3]triazol-1-yl)(4-chlorophenyl)methanone} (2a) $)^{27,40}$

White solid, yield $0.230 \mathrm{~g}(70 \%) ; \mathrm{mp} 134-137^{\circ} \mathrm{C} ; R_{f}=0.5$ (10\% ethyl acetate $/ n$-hexane).

${ }^{1} \mathrm{H}$ NMR $\left(500 \mathrm{MHz}, \mathrm{CDCl}_{3}\right): \delta=8.38(\mathrm{~d}, J=9.0 \mathrm{~Hz}, 1 \mathrm{H}), 8.22-8.17(\mathrm{~m}, 3$ $\mathrm{H}), 7.73-7.70(\mathrm{~m}, 1 \mathrm{H}), 7.57-7.55(\mathrm{~m}, 3 \mathrm{H}) \mathrm{ppm}$.
${ }^{13} \mathrm{C}$ NMR $\left(125 \mathrm{MHz}, \mathrm{CDCl}_{3}\right): \delta=165.5,145.7,140.4,133.1,132.2$, $130.5,129.7,128.8,126.4,120.2,114.7 \mathrm{ppm}$.

\section{(1H-Benzo[d][1,2,3]triazol-1-yl)(2-chlorophenyl)methanone} $(\mathbf{2 b})^{27}$

White solid, yield $0.223 \mathrm{~g}(68 \%) ; \mathrm{mp} 80-84^{\circ} \mathrm{C} ; R_{f}=0.5$ (5\% ethyl acetate/n-hexane).

${ }^{1} \mathrm{H} \mathrm{NMR}\left(500 \mathrm{MHz}, \mathrm{CDCl}_{3}\right): \delta=8.39(\mathrm{~d}, J=8.0 \mathrm{~Hz}, 1 \mathrm{H}), 8.13(\mathrm{~d}, J=9.5$ $\mathrm{Hz}, 1 \mathrm{H}), 7.71(\mathrm{t}, J=8.0 \mathrm{~Hz}, 1 \mathrm{H}), 7.65(\mathrm{~d}, J=7.0 \mathrm{~Hz}, 1 \mathrm{H}), 7.56-7.52(\mathrm{~m}$, $3 \mathrm{H}), 7.45-7.42(\mathrm{~m}, 1 \mathrm{H}) \mathrm{ppm}$.

${ }^{13} \mathrm{C}$ NMR $\left(125 \mathrm{MHz}, \mathrm{CDCl}_{3}\right): \delta=165.7,146.1,132.7,132.4,132.2$, $131.1,130.5,130.05,130.02,126.6,126.5,120.2,114.3 \mathrm{ppm}$.

\section{(1H-Benzo[d][1,2,3]triazol-1-yl)(3-chlorophenyl)methanone} (2c) $)^{27,40}$

White solid, yield $0.230 \mathrm{~g}(70 \%) ; \mathrm{mp} 122-123^{\circ} \mathrm{C} . R_{f}=0.5$ (5\% ethyl acetate/ $n$-hexane).

${ }^{1} \mathrm{H} \mathrm{NMR}\left(500 \mathrm{MHz}, \mathrm{CDCl}_{3}\right): \delta=8.40(\mathrm{~d}, J=8.5 \mathrm{~Hz}, 1 \mathrm{H}), 8.20(\mathrm{t}, J=8.5$ $\mathrm{Hz}, 2 \mathrm{H}), 8.13(\mathrm{~d}, J=7.5 \mathrm{~Hz}, 1 \mathrm{H}), 7.75-7.72(\mathrm{~m}, 1 \mathrm{H}), 7.67(\mathrm{~d}, J=7.5 \mathrm{~Hz}$, $1 \mathrm{H}), 7.59-7.51(\mathrm{~m}, 2 \mathrm{H}) \mathrm{ppm}$.

${ }^{13} \mathrm{C}$ NMR $\left(125 \mathrm{MHz}, \mathrm{CDCl}_{3}\right): \delta=165.4,145.8,134.6,133.6,133.1$, $132.2,131.5,130.6,129.8,129.7,126.5,120.3,114.7 \mathrm{ppm}$.

\section{(1H-Benzo[d][1,2,3]triazol-1-yl)(2,3-dichlorophenyl)methanone} $(\mathbf{2 d})^{27}$

White solid, yield $0.173 \mathrm{~g}(57 \%) ; \mathrm{mp} 135-136^{\circ} \mathrm{C} ; R_{f}=0.55$ (15\% ethyl acetate $/ n$-hexane).

${ }^{1} \mathrm{H}$ NMR $\left(500 \mathrm{MHz}, \mathrm{CDCl}_{3}\right): \delta=8.41(\mathrm{~d}, J=8.0 \mathrm{~Hz}, 1 \mathrm{H}), 8.17(\mathrm{~d}, J=9.0$ $\mathrm{Hz}, 1 \mathrm{H}), 7.77-7.70$ (m, $2 \mathrm{H}$ ), 7.60-7.58 (m, $1 \mathrm{H}), 7.53$ (d, J = $6.5 \mathrm{~Hz}, 1$ $\mathrm{H}), 7.41(\mathrm{t}, J=8.0 \mathrm{~Hz}, 1 \mathrm{H}) \mathrm{ppm}$.

${ }^{13} \mathrm{C}$ NMR $\left(125 \mathrm{MHz}, \mathrm{CDCl}_{3}\right): \delta=165.0,146.2,135.0,134.1,133.0$, $131.1,130.8,130.6,127.7,127.5,126.8,120.5,114.4 \mathrm{ppm}$.

\section{(1H-Benzo[d] [1,2,3]triazol-1-yl)(4-bromophenyl)methanone} $(2 \mathbf{e})^{27}$

Off-white solid, yield $0.204 \mathrm{~g}(68 \%) ; \mathrm{mp} 130-135^{\circ} \mathrm{C} ; R_{f}=0.5$ (5\% ethyl acetate/n-hexane).

${ }^{1} \mathrm{H}$ NMR $\left(500 \mathrm{MHz}, \mathrm{CDCl}_{3}\right): \delta=8.38(\mathrm{~d}, J=8.0 \mathrm{~Hz}, 1 \mathrm{H}), 8.17-8.10(\mathrm{~m}, 3$ $\mathrm{H}), 7.73-7.69(\mathrm{~m}, 3 \mathrm{H}), 7.56(\mathrm{t}, J=8.0 \mathrm{~Hz}, 1 \mathrm{H}), 7.53(\mathrm{~d}, J=6.5 \mathrm{~Hz}, 1 \mathrm{H})$, $7.41(\mathrm{t}, J=8.0 \mathrm{~Hz}, 1 \mathrm{H}) \mathrm{ppm}$.

${ }^{13} \mathrm{C}$ NMR $\left(125 \mathrm{MHz}, \mathrm{CDCl}_{3}\right): \delta=166.0,145.7,133.4,132.5,132.1$, $130.8,130.4,129.4,126.7,120.5,115.0 \mathrm{ppm}$.

\section{(1H-Benzo[d][1,2,3]triazol-1-yl)(3-bromophenyl)methanone} $(\mathbf{2 f})^{27}$

White solid, yield $0.195 \mathrm{~g}(65 \%) ; \mathrm{mp} 160-161{ }^{\circ} \mathrm{C} ; R_{f}=0.6$ (5\% ethyl acetate $/ n$-hexane).

${ }^{1} \mathrm{H}$ NMR $\left(500 \mathrm{MHz}, \mathrm{CDCl}_{3}\right): \delta=8.38-8.35(\mathrm{~m}, 2 \mathrm{H}), 8.18-8.16(\mathrm{~m}, 2 \mathrm{H})$, $7.81(\mathrm{~d}, J=8.5 \mathrm{~Hz}, 1 \mathrm{H}), 7.73-7.70(\mathrm{~m}, 1 \mathrm{H}), 7.56(\mathrm{t}, J=7.5 \mathrm{~Hz}, 1 \mathrm{H})$, 7.47-7.44 (m, $1 \mathrm{H}) \mathrm{ppm}$.

${ }^{13} \mathrm{C}$ NMR $\left(125 \mathrm{MHz}, \mathrm{CDCl}_{3}\right): \delta=165.3,145.8,136.6,134.4,133.3$, $132.2,130.7,130.3,130.0,126.6,122.5,120.3,114.8 \mathrm{ppm}$.

(1H-Benzo[d][1,2,3]triazol-1-yl)(2-bromophenyl)methanone $(2 \mathrm{~g})^{16}$

White solid, yield $0.201 \mathrm{~g}(67 \%) ; R_{f}=0.7$ (5\% ethyl acetate $/ n$-hexane). 
${ }^{1} \mathrm{H}$ NMR $\left(500 \mathrm{MHz}, \mathrm{CDCl}_{3}\right): \delta=8.41(\mathrm{~d}, J=8.5 \mathrm{~Hz}, 1 \mathrm{H}), 8.16(\mathrm{~d}, J=8.0$ $\mathrm{Hz}, 1 \mathrm{H}), 7.75-7.71$ (m, $2 \mathrm{H})$, 7.62-7.55 (m, $2 \mathrm{H})$, 7.51-7.45 (m, $2 \mathrm{H}$ ) ppm.

${ }^{13} \mathrm{C}$ NMR $\left(125 \mathrm{MHz}, \mathrm{CDCl}_{3}\right): \delta=166.4,146.2,135.0,133.2,132.5$, $131.2,130.6,130.0,127.2,126.6,120.5,120.3,114.4 \mathrm{ppm}$.

\section{(1H-Benzo[d][1,2,3]triazol-1-yl)(2,5-dibromophenyl)methanone $(\mathbf{2 h})^{18}$}

White solid, yield $0.163 \mathrm{~g}(60 \%) ; \mathrm{mp} 163-165^{\circ} \mathrm{C} ; R_{\mathrm{f}}=0.5$ (10\% ethyl acetate/ $n$-hexane).

${ }^{1} \mathrm{H}$ NMR $\left(500 \mathrm{MHz}, \mathrm{CDCl}_{3}\right): \delta=8.39(\mathrm{~d}, J=8.5 \mathrm{~Hz}, 1 \mathrm{H}), 8.17(\mathrm{~d}, J=8.5$ $\mathrm{Hz}, 1 \mathrm{H}), 7.76-7.72$ ( $\mathrm{m}, 2 \mathrm{H}), 7.60-7.57$ ( $\mathrm{m}, 3 \mathrm{H}) \mathrm{ppm}$.

${ }^{13} \mathrm{C}$ NMR $\left(125 \mathrm{MHz}, \mathrm{CDCl}_{3}\right): \delta=164.9,146.3,136.7,135.4,134.6$, $132.6,131.1,130.8,126.8,121.2,120.5,119.1,114.3 \mathrm{ppm}$.

\section{(1H-Benzo[d][1,2,3]triazol-1-yl)(3-fluorophenyl)methanone} (2i) $)^{16,40}$

White solid, yield $0.196 \mathrm{~g} \mathrm{(57 \% );} \mathrm{mp} \mathrm{100-103}{ }^{\circ} \mathrm{C} ; R_{f}=0.5$ (5\% ethyl acetate $/ n$-hexane).

${ }^{1} \mathrm{H}$ NMR $\left(500 \mathrm{MHz}, \mathrm{CDCl}_{3}\right): \delta=8.39(\mathrm{~d}, J=7.5 \mathrm{~Hz}, 1 \mathrm{H}), 8.18(\mathrm{~d}, J=8.5$ $\mathrm{Hz}, 1 \mathrm{H}), 8.05(\mathrm{~d}, J=8.5 \mathrm{~Hz}, 1 \mathrm{H}), 7.95(\mathrm{~d}, J=9.5 \mathrm{~Hz}, 1 \mathrm{H}), 7.74-7.71(\mathrm{~m}$, $1 \mathrm{H}), 7.59-7.54(\mathrm{~m}, 2 \mathrm{H}), 7.42-7.39(\mathrm{~m}, 1 \mathrm{H}) \mathrm{ppm}$.

${ }^{13} \mathrm{C}$ NMR $\left(125 \mathrm{MHz}, \mathrm{CDCl}_{3}\right): \delta=165.4,163.3,161.3,145.8,133.4(\mathrm{~d}$, $\left.J_{\mathrm{C}-\mathrm{F}}=31.9 \mathrm{~Hz}\right), 132.3,130.7,130.2\left(\mathrm{~d}, J_{\mathrm{C}-\mathrm{F}}=26.8 \mathrm{~Hz}\right), 127.6\left(\mathrm{~d}, J_{\mathrm{C}-\mathrm{F}}=\right.$ $10.8 \mathrm{~Hz}$ ), 126.6, 120.8 (d, $\left.J_{\mathrm{C}-\mathrm{F}}=81.3 \mathrm{~Hz}\right), 120.4,118.8,118.6,114.8$ ppm.

\section{(1H-Benzo[d][1,2,3]triazol-1-yl)(2-fluorophenyl)methanone (2j) ${ }^{18}$} White solid, yield $0.179 \mathrm{~g}(52 \%) ; \mathrm{mp} 96-98{ }^{\circ} \mathrm{C} ; R_{f}=0.5$ (5\% ethyl acetate/ $n$-hexane).

${ }^{1} \mathrm{H} \mathrm{NMR}\left(500 \mathrm{MHz}, \mathrm{CDCl}_{3}\right): \delta=8.38(\mathrm{~d}, J=7.5 \mathrm{~Hz}, 1 \mathrm{H}), 8.15(\mathrm{~d}, J=8.5$ $\mathrm{Hz}, 1 \mathrm{H}), 7.78(\mathrm{t}, J=6.5 \mathrm{~Hz}, 1 \mathrm{H}), 7.73-7.70(\mathrm{~m}, 1 \mathrm{H}), 7.65-7.61(\mathrm{dd}, J=$ 6.5, 7.0 Hz, $1 \mathrm{H}), 7.55(\mathrm{t}, J=7.5 \mathrm{~Hz} 1 \mathrm{H}), 7.35-7.32(\mathrm{~m}, 1 \mathrm{H}), 7.26-7.23$ (m, $1 \mathrm{H}) \mathrm{ppm}$.

${ }^{13} \mathrm{C}$ NMR $\left(125 \mathrm{MHz}, \mathrm{CDCl}_{3}\right): \delta=164.2,161.4,159.3,146.1,134.5(\mathrm{~d}$, $\left.J_{\mathrm{C}-\mathrm{F}}=31.9 \mathrm{~Hz}\right), 131.3\left(\mathrm{~d}, J_{\mathrm{C}-\mathrm{F}}=94.5 \mathrm{~Hz}\right), 130.5,126.5,124.2\left(\mathrm{~d}, J_{\mathrm{C}-\mathrm{F}}=8.9\right.$ $\mathrm{Hz}), 121.4\left(\mathrm{~d}, J_{\mathrm{C}-\mathrm{F}}=49.3 \mathrm{~Hz}\right), 120.3,116.6,116.4,114.4 \mathrm{ppm}$.

\section{3-(1H-Benzo[d][1,2,3]triazol-1-carbonyl)benzonitrile (2k)}

White solid, yield $0.209 \mathrm{~g}(62 \%) ; \mathrm{mp} \geq 180{ }^{\circ} \mathrm{C} ; R_{f}=0.3$ (10\% ethyl acetate/n-hexane).

$\left.{ }^{1} \mathrm{H} \mathrm{NMR} \mathrm{(500} \mathrm{MHz,} \mathrm{CDCl}\right)$ ): $\delta=8.55(\mathrm{~s}, 1 \mathrm{H}), 8.48(\mathrm{~d}, J=8.0 \mathrm{~Hz} 1 \mathrm{H}$ ), $8.41(\mathrm{~d}, J=9.5 \mathrm{~Hz}, 1 \mathrm{H}), 8.20(\mathrm{~d}, J=8.0 \mathrm{~Hz} 1 \mathrm{H}), 7.97(\mathrm{~d}, J=8.0 \mathrm{~Hz}, 1 \mathrm{H})$, 7.77-7.72 (m, $2 \mathrm{H}), 7.62-7.58(\mathrm{~m}, 1 \mathrm{H}) \mathrm{ppm}$.

${ }^{13} \mathrm{C}$ NMR $\left(125 \mathrm{MHz}, \mathrm{CDCl}_{3}\right): \delta=164.6,145.8,136.4,135.9,132.7$, 132.0, 130.9, 129.4, 126.8, 120.4, 117.6, 114.7, $113.1 \mathrm{ppm}$.

HRMS (ESI+): $m / z\left[\mathrm{M}+\mathrm{NH}_{4}^{+}\right]$calcd for $\mathrm{C}_{14} \mathrm{H}_{12} \mathrm{~N}_{5} \mathrm{O}^{+}: 266.1036$; found: 266.9487.

\section{(1H-Benzo[d][1,2,3]triazol-1-yl)(2-iodophenyl)methanone (21) ${ }^{26,27}$} White solid, yield $0.199 \mathrm{~g}(71 \%) ; \mathrm{mp} 80-83^{\circ} \mathrm{C} ; R_{f}=0.6$ (10\% ethyl acetate/n-hexane).

${ }^{1} \mathrm{H} \mathrm{NMR}\left(500 \mathrm{MHz}, \mathrm{CDCl}_{3}\right): \delta=8.40(\mathrm{~d}, J=7.5 \mathrm{~Hz}, 1 \mathrm{H}), 8.16(\mathrm{~d}, J=8.0$ $\mathrm{Hz}, 1 \mathrm{H}), 7.97(\mathrm{~d}, J=8.0 \mathrm{~Hz}, 1 \mathrm{H}), 7.75-7.72(\mathrm{~m}, 1 \mathrm{H}), 7.58-7.51(\mathrm{~m}, 3$ $\mathrm{H}), 7.30-7.26$ ( $\mathrm{m}, 1 \mathrm{H}) \mathrm{ppm}$.

${ }^{13} \mathrm{C}$ NMR $\left(125 \mathrm{MHz}, \mathrm{CDCl}_{3}\right): \delta=167.5,146.2,139.6,138.8,132.3$, $131.3,130.6,129.8,127.7,126.6,120.3,114.4,93.3$ ppm.
(1H-Benzo[d][1,2,3]triazol-1-yl)(m-tolyl)methanone (2m) $)^{16,40}$ White solid, yield $0.243 \mathrm{~g}$ (70\%); $\mathrm{mp} 65-66{ }^{\circ} \mathrm{C} ; R_{f}=0.5$ (5\% ethyl acetate/n-hexane).

${ }^{1} \mathrm{H}$ NMR $\left(500 \mathrm{MHz}, \mathrm{CDCl}_{3}\right): \delta=8.38(\mathrm{~d}, J=8.0 \mathrm{~Hz}, 1 \mathrm{H}), 8.17(\mathrm{~d}, J=8.5$ $\mathrm{Hz}, 1 \mathrm{H}), 8.01(\mathrm{~d}, J=6.5 \mathrm{~Hz}, 2 \mathrm{H}), 7.72-7.69(\mathrm{~m}, 1 \mathrm{H}), 7.56-7.53(\mathrm{~m}, 1$ H), 7.51-7.44 (m, $2 \mathrm{H}), 2.48$ (s, $3 \mathrm{H}) \mathrm{ppm}$.

${ }^{13} \mathrm{C}$ NMR $\left(125 \mathrm{MHz}, \mathrm{CDCl}_{3}\right): \delta=166.9,145.7,138.3,134.4,132.3$, $132.0,131.4,130.3,128.9,128.2,126.2,120.1,114.7,21.3$ ppm.

\section{(1H-Benzo[d][1,2,3]triazol-1-yl)(3,5-dimethylphenyl)methanone} $(2 n)^{18}$

White solid, yield $0.230 \mathrm{~g}(69 \%) ; \mathrm{mp} 68-70{ }^{\circ} \mathrm{C} ; R_{f}=0.5$ (5\% ethyl acetate/n-hexane).

${ }^{1} \mathrm{H} \mathrm{NMR}\left(500 \mathrm{MHz}, \mathrm{CDCl}_{3}\right): \delta=8.37(\mathrm{~d}, J=8.0 \mathrm{~Hz}, 1 \mathrm{H}), 8.16(\mathrm{~d}, J=8.0$ $\mathrm{Hz}, 1 \mathrm{H}), 7.78$ (s, $2 \mathrm{H}$ ), 7.72-7.67 ( $\mathrm{m}, 1 \mathrm{H}), 7.55-7.52$ ( $\mathrm{m}, 1 \mathrm{H}), 7.31$ (s, 1 H) ppm.

${ }^{13} \mathrm{C}$ NMR $\left(125 \mathrm{MHz}, \mathrm{CDCl}_{3}\right): \delta=167.1,145.6,138.1,135.4,132.3$, $131.3,130.2,129.2,127.8,126.2,125.9,120.0,114.7,21.2 \mathrm{ppm}$.

\section{(1H-Benzo[d][1,2,3]triazol-1-yl)(2-methoxyphenyl)methanone} $(20)^{27}$

White solid, yield $0.236 \mathrm{~g}(71 \%) ; \mathrm{mp} 92-94{ }^{\circ} \mathrm{C} ; R_{f}=0.5$ (5\% ethyl acetate/n-hexane).

${ }^{1} \mathrm{H}$ NMR $\left(500 \mathrm{MHz}, \mathrm{CDCl}_{3}\right): \delta=8.39(\mathrm{~d}, J=8.5 \mathrm{~Hz}, 1 \mathrm{H}), 8.13(\mathrm{~d}, J=7.5$ $\mathrm{Hz}, 1 \mathrm{H}), 7.71-7.68$ ( $\mathrm{m}, 1 \mathrm{H}), 7.62-7.51(\mathrm{~m}, 3 \mathrm{H}), 7.13-7.06(\mathrm{~m}, 2 \mathrm{H})$, 3.77 (s, $3 \mathrm{H}) \mathrm{ppm}$.

${ }^{13} \mathrm{C}$ NMR $\left(125 \mathrm{MHz}, \mathrm{CDCl}_{3}\right): \delta=166.9,157.8,146.0,133.5,131.4$, $130.2,130.1,126.1,122.7,120.4,120.0,114.4,111.4,55.7$ ppm.

\section{(1H-Benzo[d][1,2,3]triazol-1-yl)(phenyl)methanone (2p) $)^{26}$}

White solid, yield $0.274 \mathrm{~g}$ (75\%); mp $110-112{ }^{\circ} \mathrm{C} ; R_{f}=0.6$ (5\% ethyl acetate/n-hexane).

${ }^{1} \mathrm{H} \mathrm{NMR}\left(500 \mathrm{MHz}, \mathrm{CDCl}_{3}\right): \delta=8.40(\mathrm{~d}, J=8.0 \mathrm{~Hz}, 1 \mathrm{H}), 8.23-8.17(\mathrm{~m}, 3$ H), 7.73-7.68 (dd, $J=15.5,8.0 \mathrm{~Hz}, 2 \mathrm{H}), 7.60-7.54(\mathrm{~m}, 3 \mathrm{H}) \mathrm{ppm}$.

${ }^{13} \mathrm{C}$ NMR $\left(125 \mathrm{MHz}, \mathrm{CDCl}_{3}\right): \delta=166.7,145.7,133.6,132.3,131.7$, $131.4,130.4,128.4,126.3,120.1,114.7 \mathrm{ppm}$.

(1H-Benzo[d][1,2,3]triazol-1-yl)(napthalen-1-yl)methanone (2q) ${ }^{27}$ White solid, yield $0.253 \mathrm{~g}$ (80\%); $\mathrm{mp} 137-140{ }^{\circ} \mathrm{C} ; R_{f}=0.7$ (5\% ethyl acetate/n-hexane).

${ }^{1} \mathrm{H}$ NMR $\left(500 \mathrm{MHz}, \mathrm{CDCl}_{3}\right): \delta=8.50(\mathrm{~d}, J=8.0 \mathrm{~Hz}, 1 \mathrm{H}), 8.19-8.12(\mathrm{~m}, 3$ H), 7.98-7.94 (m, $2 \mathrm{H}), 7.81-7.75$ ( $\mathrm{m}, 1 \mathrm{H}), 7.63-7.57$ (m, $4 \mathrm{H}$ ) ppm.

${ }^{13} \mathrm{C}$ NMR $\left(125 \mathrm{MHz}, \mathrm{CDCl}_{3}\right): \delta=167.6,146.1,133.5,132.9,132.0$, $131.0,130.4,130.1,129.3,128.7,127.9,126.7,126.4,124.7,124.2$, 120.3, $114.7 \mathrm{ppm}$.

(1H-Benzo[d][1,2,3]triazol-1-yl)(furan-2-yl)methanone (2r) ${ }^{26}$ White solid, yield $0.239 \mathrm{~g}(63 \%) ; \mathrm{mp} 168-170{ }^{\circ} \mathrm{C} ; R_{\mathrm{f}}=0.8$ (10\% ethyl acetate $/ n$-hexane).

${ }^{1} \mathrm{H} \mathrm{NMR}\left(500 \mathrm{MHz}, \mathrm{CDCl}_{3}\right): \delta=8.41(\mathrm{~d}, J=8.5 \mathrm{~Hz}, 1 \mathrm{H}), 8.17-8.14(\mathrm{~m}, 2$ H), 7.87-7.85 (m, $1 \mathrm{H}), 7.70-7.67$ ( $\mathrm{m}, 1 \mathrm{H}), 7.55-7.52$ (m, $1 \mathrm{H}), 6.73-$ $6.72(\mathrm{~m}, 1 \mathrm{H}) \mathrm{ppm}$.

${ }^{13} \mathrm{C}$ NMR $\left(125 \mathrm{MHz}, \mathrm{CDCl}_{3}\right): \delta=155.1,149.0,145.6,144.7,132.2$, 130.6, 126.4, 124.8, 120.3, 114.8, 113.0 ppm. 


\section{(1H-Benzo[d][1,2,3]triazol-1-yl)(trifluoromethyl)phenyl)metha-} none (2s) $)^{19,27}$

White solid, yield $0.150 \mathrm{~g}(49 \%) ; \mathrm{mp} 55-58{ }^{\circ} \mathrm{C} ; R_{f}=0.7$ (10\% ethyl acetate/n-hexane).

${ }^{1} \mathrm{H} \mathrm{NMR}\left(500 \mathrm{MHz}, \mathrm{CDCl}_{3}\right): \delta=8.49(\mathrm{~s}, 1 \mathrm{H}), 8.44(\mathrm{~d}, J=7.5 \mathrm{~Hz}, 1 \mathrm{H})$, $8.38(\mathrm{~d}, J=9.0 \mathrm{~Hz}, 1 \mathrm{H}), 8.17(\mathrm{~d}, J=8.5 \mathrm{~Hz}, 1 \mathrm{H}), 7.95(\mathrm{~d}, J=7.5 \mathrm{~Hz}, 1 \mathrm{H})$, 7.74-7.71 (m, 2 H), 7.58-7.55 (m, $1 \mathrm{H}) \mathrm{ppm}$.

${ }^{13} \mathrm{C}$ NMR $\left(125 \mathrm{MHz}, \mathrm{CDCl}_{3}\right): \delta=165.3,145.7,134.8,132.1\left(\mathrm{~d}, J_{\mathrm{C}-\mathrm{F}}=87.4\right.$ $\mathrm{Hz}), 131.1\left(\mathrm{~d}, J_{\mathrm{C}-\mathrm{F}}=119.0 \mathrm{~Hz}\right), 130.7,130.04\left(\mathrm{~d}, J_{\mathrm{C}-\mathrm{F}}=12.7 \mathrm{~Hz}\right), 129.0$, $128.5\left(\mathrm{~d}, J_{\mathrm{C}-\mathrm{F}}=12.2 \mathrm{~Hz}\right), 126.6,124.5,120.3,114.7 \mathrm{ppm}$.

\section{(E)-1-(1H-Benzo[d][1,2,3]triazol-1-yl)-3-phenylprop-2-en-1-one} $(2 \mathrm{t})^{41}$

White solid, yield $0.198 \mathrm{~g}$ (59\%); mp $150-151{ }^{\circ} \mathrm{C} ; R_{f}=0.5$ (50\% ethyl acetate/n-hexane).

${ }^{1} \mathrm{H}$ NMR $\left(500 \mathrm{MHz}, \mathrm{CDCl}_{3}\right): \delta=8.43(\mathrm{~d}, J=8.0 \mathrm{~Hz}, 1 \mathrm{H}), 8.16-8.15(\mathrm{~m}, 3$ H), 7.77-7.75 (m, $2 \mathrm{H}), 7.69(\mathrm{t}, J=8.0 \mathrm{~Hz}, 1 \mathrm{H}), 7.55-7.52(\mathrm{~m}, 1 \mathrm{H})$, 7.48-7.47 (m, $3 \mathrm{H}) \mathrm{ppm}$,

${ }^{13} \mathrm{C}$ NMR $\left(125 \mathrm{MHz}, \mathrm{CDCl}_{3}\right): \delta=163.9,148.7,146.3,134.1,131.4$, $130.3,129.1,129.0,126.2,120.1,116.0,114.8 \mathrm{ppm}$.

\section{1-(1H-Benzo[d] [1,2,3]triazol-1-yl)heptan-1-one (3a) $)^{42}$}

Light yellow semisolid, yield $0.241 \mathrm{~g}(68 \%) ; R_{f}=0.5$ (5\% ethyl acetate/n-hexane).

${ }^{1} \mathrm{H} \mathrm{NMR}\left(500 \mathrm{MHz}, \mathrm{CDCl}_{3}\right): \delta=8.28(\mathrm{~d}, J=8.0 \mathrm{~Hz}, 1 \mathrm{H}), 8.10(\mathrm{~d}, J=8.0$ $\mathrm{Hz}, 1 \mathrm{H}), 7.63(\mathrm{t}, J=8.0 \mathrm{~Hz}, 1 \mathrm{H}), 7.50-7.47(\mathrm{~m}, 1 \mathrm{H}), 3.42-3.39(\mathrm{~m}, 2$ H), 1.92-1.86 (m, 2 H), 1.50-1.44 (m, 2 H), 1.38-1.31 (m, 4 H), 0.90$0.88(\mathrm{~m}, 3 \mathrm{H}) \mathrm{ppm}$.

${ }^{13} \mathrm{C}$ NMR $\left(125 \mathrm{MHz}, \mathrm{CDCl}_{3}\right): \delta=172.6,146.0,131.0,130.2,125.9$, $120.0,114.3,35.4,31.4,28.7,24.3,22.4,13.9 \mathrm{ppm}$.

\section{1-(1H-Benzo[d][1,2,3]triazol-1-yl)decan-1-one (3b)}

Colorless oil, yield $0.199 \mathrm{~g}$ (63\%); $R_{f}=0.5$ (5\% ethyl acetate $/ n$-hexane). ${ }^{1} \mathrm{H}$ NMR $\left(500 \mathrm{MHz}, \mathrm{CDCl}_{3}\right): \delta=8.28(\mathrm{~d}, J=9.0 \mathrm{~Hz}, 1 \mathrm{H}), 8.11(\mathrm{~d}, J=8.0$ $\mathrm{Hz}, 1 \mathrm{H}), 7.63(\mathrm{t}, J=8.0 \mathrm{~Hz}, 1 \mathrm{H}), 7.48(\mathrm{t}, J=8.0 \mathrm{~Hz}, 1 \mathrm{H}), 3.40(\mathrm{t}, J=8.0$ $\mathrm{Hz}, 2 \mathrm{H}), 1.92-1.85(\mathrm{~m}, 2 \mathrm{H}), 1.49-1.43(\mathrm{~m}, 2 \mathrm{H}), 1.39-1.25(\mathrm{~m}, 10 \mathrm{H})$, $0.86(\mathrm{t}, J=6.5 \mathrm{~Hz}, 3 \mathrm{H}) \mathrm{ppm}$.

${ }^{13} \mathrm{C}$ NMR $\left(125 \mathrm{MHz}, \mathrm{CDCl}_{3}\right): \delta=172.6,146.1,131,0,130.2,125.9,120.0$, $114.4,35.4,31.8,29.3,29.25,29.20,29.0,24.4,22.6,14.0 \mathrm{ppm}$.

HRMS (ESI+): $m / z$ [M + Na] calcd for $\mathrm{C}_{16} \mathrm{H}_{23} \mathrm{~N}_{3} \mathrm{NaO}$ : 296.1739; found: 296.1745 .

\section{1-(1H-Benzo[d][1,2,3]triazol-1-yl)tetradecan-1-one (3c) $)^{43}$}

Yellowish solid, yield $0.158 \mathrm{~g}(55 \%) ; \mathrm{mp} 40-42{ }^{\circ} \mathrm{C} ; R_{\mathrm{f}}=0.5$ (5\% ethyl acetate/ $n$-hexane);

${ }^{1} \mathrm{H}$ NMR $\left(500 \mathrm{MHz}, \mathrm{CDCl}_{3}\right): \delta=8.30(\mathrm{~d}, J=8.0 \mathrm{~Hz}, 1 \mathrm{H}), 8.12(\mathrm{~d}, J=8.0$ $\mathrm{Hz}, 1 \mathrm{H}), 7.65(\mathrm{t}, J=8.0 \mathrm{~Hz}, 1 \mathrm{H}), 7.50(\mathrm{t}, J=8.0 \mathrm{~Hz}, 1 \mathrm{H}), 3.43-3.40(\mathrm{~m}$, $2 \mathrm{H}), 1.94-1.87$ (m, $2 \mathrm{H}), 1.51-1.45(\mathrm{~m}, 2 \mathrm{H}), 1.39-1.25(\mathrm{~m}, 18 \mathrm{H})$, $0.89-0.86(\mathrm{~m}, 3 \mathrm{H}) \mathrm{ppm}$.

${ }^{13} \mathrm{C}$ NMR $\left(125 \mathrm{MHz}, \mathrm{CDCl}_{3}\right): \delta=172.7,146.2,131.1,130.3,126.0$, 120.1, 114.4, 35.5, 31.9, 29.65, 29.62, 29.5, 29.4, 29.3, 29.2, 29.1, 29.0, 24.4, 22.6, $14.1 \mathrm{ppm}$.

\section{1-(1H-Benzo[d] [1,2,3]triazol-1-yl)hexadecan-1-one (3d $)^{44}$}

White solid, yield $0.136 \mathrm{~g}(49 \%) ; \mathrm{mp} 48-50{ }^{\circ} \mathrm{C} ; R_{f}=0.5$ (5\% ethyl acetate/n-hexane).
${ }^{1} \mathrm{H}$ NMR $\left(500 \mathrm{MHz}, \mathrm{CDCl}_{3}\right): \delta=8.30(\mathrm{~d}, J=8.0 \mathrm{~Hz}, 1 \mathrm{H}), 8.12(\mathrm{~d}, J=8.0$ $\mathrm{Hz}, 1 \mathrm{H}), 7.66-7.63(\mathrm{~m}, 1 \mathrm{H}), 7.50(\mathrm{t}, J=8.0 \mathrm{~Hz}, 1 \mathrm{H}), 3.43-3.40(\mathrm{~m}, 2$ H), 1.93-1.87 (m, 2 H), 1.49-1.44 (m, 2 H), 1.40-1.25 (m, 22 H), 0.88$0.86(\mathrm{~m}, 3 \mathrm{H}) \mathrm{ppm}$.

${ }^{13} \mathrm{C}$ NMR $\left(125 \mathrm{MHz}, \mathrm{CDCl}_{3}\right): \delta=172.7,146.1,131.1,130.3,126.0$, $120.1,114.4,35.5,31.9,29.6,29.5,29.4,29.3,29.2,29.1,29.0,24.4$, 22.6, $14.1 \mathrm{ppm}$.

HRMS (ESI+): $m / z[M+H]$ calcd for $\mathrm{C}_{22} \mathrm{H}_{36} \mathrm{~N}_{3} \mathrm{O}$ : 358.2858; found: 358.2875 .

\section{Conflict of Interest}

The authors declare no conflict of interest.

\section{Funding Information}

The author sincerely thanks the Council of Scientific and Industrial Research (CSIR), New Delhi (Grant Number 02(0345)/19/EMR-II) and Banaras Hindu University (IoE grant) for funding. MSY and MKJ acknowledge the Council of Scientific and Industrial Research (CSIR) for SRF Fellowships and SK for a Junior Research Fellowship. The authors also thank CISC-Banaras Hindu University, Varanasi.

\section{Supporting Information}

Supporting information for this article is available online at https://doi.org/10.1055/a-1656-7293. Included are copies of ${ }^{1} \mathrm{H}$ and ${ }^{13} \mathrm{C}$ NMR for all the synthesized ureas and their derivatives and thiocarbamates for this article.

\section{References}

(1) Katritzky, A. R.; Lan, X.; Yang, J. Z.; Denisko, O. V. Chem. Rev. 1998, 98, 409.

(2) Briguglio, I.; Piras, S.; Corona, P.; Gavini, E.; Nieddu, M.; Boatto, G.; Carta, A. Eur. J. Med. Chem. 2015, 97, 612.

(3) Katritzky, A. R.; Rachwal, S. Chem. Rev. 2010, 110, 1564.

(4) (a) Kale, R. R.; Prasad, V.; Mohapatra, P. P.; Tiwari, V. K. Monatsh. Chem. 2010, 141, 1159. (b) Yu, J.; Singh, A. S.; Yan, G.; Yu, J.; Tiwari, V. K. Synthesis 2020, 52, 3781.

(5) Singh, M.; Singh, A. S.; Mishra, N.; Agrahari, A. K.; Tiwari, V. K. ACS Omega 2019, 4, 2418.

(6) (a) Katritzky, A. R.; Rogovoy, B. V.; Kirichenko, N.; Vvedensky, V. Bioorg. Med. Chem. Lett. 2002, 12, 1809. (b) Katritzky, A. R.; Suzuki, K.; Singh, S. K.; He, H.-Y. J. Org. Chem. 2003, 68, 5720. (c) Katritzky, A. R.; Singh, S. K.; Cai, C.; Bobrov, S. J. Org. Chem. 2006, 71, 3364. (d) Kale, R. R.; Prasad, V.; Tiwari, V. K. Lett. Org. Chem. 2010, 7, 136.

(7) (a) Zhou, G.; Lim, D.; Fang, F.; Coltart, D. M. Synthesis 2009, 3350. (b) Xia, Z.; Lv, X.; Wang, W.; Wang, X. Tetrahedron Lett. 2011, 52, 4906. (c) Katritzky, A. R.; Shestopalov, A. A.; Suzuki, K. Synthesis 2004, 1806.

(8) Katritzky, A. R.; Widyan, K.; Kirichenko, K. J. Org. Chem. 2007, $72,5802$.

(9) Gonnet, Lori.; Tintillier, T.; Venturini, N.; Konnert, L.; Hernandez, J.-F.; Lamaty, F.; Laconde, G.; Martinez, J.; Colacino, E. ACS Sustainable Chem. Eng. 2017, 5, 2936.

(10) (a) Wang, X.; Zhang, Y. Synth. Commun. 2003, 33, 2627. (b) Lim, D.; Fang, F.; Zhou, G.; Coltart, D. M. Org. Lett. 2007, 9, 4139. 
(11) Katritzky, A. R.; Abdel-Fattah, A. A. A.; Wang, M. J. Org. Chem. 2003, 68, 4932.

(12) Katritzky, A. R.; Abdel-Fattah, A. A. A.; Wang, M. J. Org. Chem. 2003, 68, 1443.

(13) Katritzky, A. R.; Cai, C.; Suzuki, K.; Singh, S. K. J. Org. Chem. 2004, $69,811$.

(14) (a) Wang, X.; Wang, W.; Wen, Y.; He, L.; Zhu, X. Synthesis 2008, 3223. (b) Katritzky, A. R.; Suzuki, K.; Wang, Z. Synlett 2005, 1656. (c) Li, J.; Sun, Y.; Chen, Z.; Su, W. Synth. Commun. 2010, 40, 3669.

(15) Singh, A. S.; Kumar, D.; Mishra, N.; Tiwari, V. K. ChemistrySelect 2017, 2224.

(16) Singh, A. S.; Mishra, N.; Kumar, D.; Tiwari, V. K. ACS Omega 2017, 2,5044 .

(17) (a) Yadav, M. S.; Singh, A. S.; Agrahari, A. K.; Mishra, N.; Tiwari, V. K. ACS Omega 2019, 4, 6681. (b) Kumar, D.; Mishra, B. B.; Tiwari, V. K. J. Org. Chem. 2014, 79, 251. (c) Kumar, D.; Mishra, A.; Mishra, B. B.; Bhattacharya, S.; Tiwari, V. K. J. Org. Chem. 2013, 78, 899. (d) Singh, A. S.; Kumar, D.; Tiwari, V. K. ChemistrySelect 2018, 3, 7809. (e) Singh, A. S.; Mishra, N.; Yadav, M. S.; Tiwari, V. K. J. Heterocycl. Chem. 2019, 56, 275.

(18) (a) Yadav, M. S.; Singh, S. K.; Agrahari, A. K.; Singh, A. S.; Tiwari, V. K. Synthesis 2021, 53, 2494. (b) Singh, A. S.; Kumar, D.; Mishra, N.; Tiwari, V. K. RSC Adv. 2016, 6, 84512. (c) Singh, A. S.; Agrahari, A. K.; Singh, S. K.; Yadav, M. S.; Tiwari, V. K. Synthesis 2019, 51, 3443.

(19) (a) Singh, A. S.; Agrahari, A. K.; Singh, M.; Mishra, N.; Tiwari, V. K. ARKIVOC 2017, $(v), 80$

(20) Agha, K. A.; Abo-Dya, N. E.; Ibrahim, T. S.; Abdel-Aal, E. H. ARKIVOC 2016, (iii), 161.

(21) Singh, M.; Agrahari, A. K.; Mishra, N.; Singh, A. S.; Tiwari, V. K. Ind. J. Heterocycl. Chem. 2018, 28, 125.

(22) Duangkamol, C.; Wangngae, S.; Pattarawarapan, M.; Phakhodee, W. Eur. J. Org. Chem. 2014, 7109.

(23) Kanışkan, N.; Kökten, S.; Celik, I. ARKIVOC 2012, (viii), 198.

(24) Wet-osot, S.; Duangkamol, C.; Pattarawarapan, M.; Phakhodee, W. Monatsh. Chem. 2015, 146, 959.

(25) (a) Katritzky, A. R.; Shobana, N.; Pernak, J.; Afridi, A. S.; Fan, W. Q. Tetrahedron 1992, 48, 7817. (b) Katritzky, A. R.; Zhang, Y.; Singh, S. K. Synthesis 2003, 2795.
(26) Singh, A. S.; Agrahari, A. K.; Mishra, N.; Singh, M.; Tiwari, V. K. Synthesis 2019, 51, 470.

(27) Singh, M.; Singh, A. S.; Mishra, N.; Agrahari, A. K.; Tiwari, V. K. Synthesis 2019, 51, 2183.

(28) Jang, D. O.; Park, D. J.; Kim, J. Tetrahedron Lett. 1999, 5323.

(29) Prusinowski, A. F.; Twumasi, R. K.; Wappes, E. A.; Nagib, D. A. J. Am. Chem. Soc. 2020, 142, 5429.

(30) Tanaka, R.; Tanimoto, I.; Kojima, M.; Yoshino, T.; Matsunaga, S. J. Org. Chem. 2019, 84, 13203.

(31) Mezailles, N.; Ricard, L.; Gagosz, F. Org. Lett. 2005, 7, 4133.

(32) Gayakhe, V.; Ardhapure, A. K.; Kapdi, A. R.; Sanghvi, Y. S.; Serrano, J. L.; García, L.; Pérez, J.; García, J.; Sánchez, G.; Fischer, C.; Schulzke, C. J. Org. Chem. 2016, 81, 2713.

(33) Agrahari, A. K.; Bose, P.; Jaiswal, M. K.; Rajkhowa, S.; Singh, A. S.; Hotha, S.; Mishra, N.; Tiwari, V. K. Chem. Rev. 2021, 12, 7638.

(34) Tiwari, V. K.; Mishra, B. B.; Mishra, K. B.; Mishra, N.; Singh, A. S.; Chen, X. Chem. Rev. 2016, 116, 3086.

(35) Agrahari, A. K.; Jaiswal, M. K.; Yadav, M. S.; Tiwari, V. K. Carbohydr. Res. 2021, 508, 108403.

(36) Agrahari, A. K.; Singh, A. S.; Mukharjee, R. K.; Tiwari, V. K. RSC Adv. 2020, 43, 31553.

(37) Schmidt, R. R.; Michel, J. Angew. Chem., Int. Ed. Engl. 1980, 19, 731.

(38) (a) Schmidt, R. R.; Michel, J. J. Carbohydr. Chem. 1985, 4, 141. (b) Schmidt, R. R.; Kinzy, W. Adv. Carbohydr. Chem. Biochem. 1994, 50, 21.

(39) Maharani, N. S.; Meador, R. I. L.; Smith, T. J.; Canarelli, S. E.; Adhikari, A. A.; Shah, J. P.; Russo, C. M.; Wallach, D. R.; Howard, K. T.; Millimaci, A. M.; Chisholm, J. D. J. Org. Chem. 2019, 84, 7871.

(40) Betori, R. C.; Miller, E. R.; Scheidt, K. A. Adv. Synth. Catal. 2017, 359, 1131.

(41) Katritzky, A. R.; Cai, C.; Singh, S. K. J. Org. Chem. 2006, 71, 3375.

(42) Wet-osot, S.; Phakhodee, W.; Pattarawarapan, M. Tetrahedron Lett. 2015, 56, 6998.

(43) Matilde, C. V.; Nagel Heinze, T. Polym. Bull. 2010, 65, 873.

(44) Pocquet, L.; Vologdin, N.; Mangiatordi, G. F.; Ciofini, I.; Nicolotti, O.; Thorimbert, S.; Salmain, M. Eur. J. Inorg. Chem. 2017, 3622. 